\title{
Turing pattern formation in the Brusselator system with nonlinear diffusion
}

\author{
G. Gambino* M.C. Lombardo ${ }^{\dagger}$ M. Sammartino ${ }^{\ddagger}$ V. Sciacca ${ }^{\S}$
}

October 25, 2013

\begin{abstract}
In this work we investigate the effect of density dependent nonlinear diffusion on pattern formation in the Brusselator system. Through linear stability analysis of the basic solution we determine the Turing and the oscillatory instability boundaries. A comparison with the classical linear diffusion shows how nonlinear diffusion favors the occurrence of Turing pattern formation. We study the process of pattern formation both in 1D and $2 \mathrm{D}$ spatial domains. Through a weakly nonlinear multiple scales analysis we derive the equations for the amplitude of the stationary patterns. The analysis of the amplitude equations shows the occurrence of a number of different phenomena, including stable supercritical and subcritical Turing patterns with multiple branches of stable solutions leading to hysteresis. Moreover we consider traveling patterning waves: when the domain size is large, the pattern forms sequentially and traveling wavefronts are the precursors to patterning. We derive the Ginzburg-Landau equation and describe the traveling front enveloping a pattern which invades the domain. We show the emergence of radially symmetric target patterns, and through a matching procedure we construct the outer amplitude equation and the inner core solution.
\end{abstract}

\section{Introduction}

The aim of this work is to describe the Turing pattern formation for the following reactiondiffusion system, introduced in [18]:

$$
\begin{aligned}
& \frac{\partial U}{\partial \tau}=D_{u} \frac{\partial}{\partial \zeta}\left(\left(\frac{U}{u_{0}}\right)^{m} \frac{\partial U}{\partial \zeta}\right)+\Gamma\left(a-(b+1) U+U^{2} V\right), \\
& \frac{\partial V}{\partial \tau}=D_{v} \frac{\partial}{\partial \zeta}\left(\left(\frac{V}{v_{0}}\right)^{n} \frac{\partial V}{\partial \zeta}\right)+\Gamma\left(b U-U^{2} V\right) .
\end{aligned}
$$

Here $U(\zeta, \tau)$ and $V(\zeta, \tau)$, with $\zeta \in[0, l]$, represent the concentrations of two chemical species, the activator and the inhibitor respectively; $a$ and $b$ are positive constants; the constant $\Gamma>0$ represents the strength of the reaction terms or, alternatively, modulates the size of the domain.

\footnotetext{
*Department of Mathematics, University of Palermo, Italy, gaetana@math.unipa.it

${ }^{\dagger}$ Department of Mathematics, University of Palermo, Italy, lombardo@math.unipa.it

${ }^{\ddagger}$ Department of Mathematics, University of Palermo, Italy, marco@math.unipa.it

$\S^{\S}$ Department of Mathematics, University of Palermo, Italy, sciacca@math.unipa.it
} 
Equations (1.1) belong to the class of reaction-diffusion systems with nonlinear diffusion. The nonlinear density-dependent diffusion present in (1.1) is such that, when $m, n>0$, the species tend to diffuse faster (when $U>u_{0}$ and $V>v_{0}$ ) or slower (when $U<u_{0}$ and $\left.V<v_{0}\right)$ than predicted by the linear classical diffusion. The coefficients $D_{u}, D_{v}>0$ are the classical diffusion coefficients and the nonnegative $u_{0}$ and $v_{0}$ are threshold concentrations which measure the strength of the interactions between the individuals of the same species. At microscopic level this kind of diffusion term can be interpreted as the result of the interaction between random walkers representing the individuals of the system; whereas classical diffusion corresponds to the case of independent random walks. In particular, the dynamics is subdiffusive as the mean square displacement of the particles $\left\langle(\Delta \zeta)^{2}\right\rangle \sim \tau^{\alpha}$, with $\alpha=1 /(2+m)<$ 1 (for the $V$ species $\alpha=1 /(2+n)$ ), see the discussion in [20].

The reaction mechanism is chosen as in the Brusselator autocatalytic system. This system is a model used to capture the qualitative behavior of cross activator-inhibitor chemical reactions: to this class belong some autocatalytic reactions such as ferrocyanide-iodate-sulphite reaction, chlorite-iodide-malonic acid (CIMA) reaction, arsenite-iodate reaction, and many enzyme catalytic reactions. For a review on the rich spatial and temporal dynamics shown by cubic-autocatalytic reaction-diffusion systems, see e.g. [13, 17.

In general one can say that models like (1.1) are believed to be relevant for autocatalytic chemical reactions occurring: a) on a binding hydrogel substrate [21, 28]; b) on surfaces [15. 31, 30, like cellular membranes, or in phenomena of industrial interest like surface electrodeposition [3] or metal catalysis [14, 23]; c) on porous media[1, 43, 44].

Recently reaction-diffusion models with concentration-dependent diffusion coefficients have attracted considerable attention in many different fields [9, 10, 26, 36, 17, 8, 32, 34, 37, 6, 24, 4]; however the effect of the anomalous diffusivity on Turing pattern of chemical and biochemical systems is not yet fully investigated, due to the presence of complicated reaction terms and related difficulties even in the linear stability analysis. As exceptions here we mention [25], where it was shown that the introduction of concentration dependence of the diffusion coefficient, due to the ionic character of the reactants, sharpens the features of the pattern, resulting in an increase of the chemical gradients of the chemicals; or the paper [31], where pattern formation in the Gray-Scott model of excitable media with diffusion coefficient linearly dependent on the concentration was studied; the mechanism responsible for the formation of the pattern was however different from Turing bifurcation; and the recent [22] where the authors consider and explore, numerically, the Lengyel-Epstein model with local concentration-dependent diffusivity. More specifically for the Brusselator system, recently, have appeared several papers considering pattern formation in presence of cross-diffusion 
[19, 41, 42] and superdiffusion due to the fractional Laplacian [12, 39, 38].

Regarding the system (1.1), in [18] the authors derived the conditions for Turing instability and showed that, differently from the standard linear diffusion case, the destabilization of the constant steady state, occurs even if the diffusion constant $D_{v}$ of the inhibitor is smaller or equal to the diffusion constant $D_{u}$ of the activator. In this paper we reconsider the linear stability analysis of the system (1.1) taking into account the fact that the Brusselator kinetics also supports Hopf bifurcation: even though the steady state is Turing unstable, whether Turing patterns form depends on the mutual location of the Hopf and the Turing instability boundaries. This will be analyzed in Section 2, where the Turing and Hopf stability boundaries will be obtained in terms of three key system parameters. This will clarify the role of nonlinear diffusion in the formation of the pattern. In Section 3 we shall perform the weakly nonlinear analysis near the onset of the Turing instability. The amplitude equation will be derived both for stationary pattern (Stuart-Landau amplitude equation) and spatially modulated pattern (real Ginzburg-Landau amplitude equation). Moreover, we shall derive the quintic Stuart-Landau equation which describes the phenomenon of hysteresis occurring in the case when the bifurcation is subcritical. Numerical simulations are performed to corroborate the predictions coming from the weakly nonlinear analysis. In Section 4 we shall focus on pattern formation in a $2 \mathrm{D}$ domain. Rolls and squares, which arise when the homogeneous steady state bifurcates at a simple eigenvalue, and mixed-mode patterns, which emerge when the eigenvalue is double and different modes interact, will be shown. Particular mixed-mode patterns are the hexagonal patterns, which appear when a resonance condition holds. The evolution system for the amplitudes of the patterns in each case will be given and discussed. The emergence of axisymmetric target patterns will then be shown and an asymptotic matching procedure will be employed to derive the appropriate amplitude equation.

We finally believe that, in the context of the previously mentioned phenomena of surface chemical reactions and chemical instabilities in porous media, the model (1.1) and the mathematical analysis presented in this paper, could be of support for the quantitative prediction of the observed dynamics and for the design of more focused experiments. For example, as discussed in [18, the analysis presented here (and in [18]) should motivate and solicit the realization of experiments devoted to the investigation of dissipative structures in open chemical reactors where porous media are employed.

\section{Linear stability analysis}

In analogy with [12], we rescale (1.1), using $U=u^{*} u, V=v^{*} v, \tau=t, \zeta=x^{*} x$, where:

$$
\begin{aligned}
u^{*} & =\left(\frac{(m+1) D_{v} u_{o}^{m}}{(n+1) D_{u} v_{0}^{n}}\right)^{\frac{1}{m+n+2}}, \quad v^{*}=\frac{1}{u^{*}}, \\
x^{*} & =\sqrt{\frac{D_{v}}{(n+1) v_{0}^{n} u^{*(n+2)}}}
\end{aligned}
$$


to obtain:

$$
\begin{aligned}
& \frac{\partial u}{\partial t}=\frac{\partial^{2}}{\partial x^{2}} u^{m+1}+\Gamma\left(Q-(b+1) u+u^{2} v\right) \\
& \frac{\partial v}{\partial t}=\frac{1}{\eta^{2}} \frac{\partial^{2}}{\partial x^{2}} v^{n+1}+\frac{\Gamma}{\eta^{2}}\left(b u-u^{2} v\right)
\end{aligned}
$$

having defined:

$$
Q=a \eta \quad \text { and } \quad \eta=1 / u^{*} .
$$

The system (2.2) is supplemented with initial data and Neumann boundary conditions. The only nontrivial homogeneous stationary solution admitted by the system $(2.2)$ is $(\bar{u}, \bar{v}) \equiv$ $(Q, b / Q)$. Through linear stability analysis one gets the following dispersion relation which gives the growth rate $\sigma$ as a function of the wavenumber $k$ :

$$
\sigma^{2}+g\left(k^{2}\right) \sigma+h\left(k^{2}\right)=0,
$$

where:

$$
\begin{aligned}
& g\left(k^{2}\right)=k^{2} \operatorname{tr}(D)-\Gamma \operatorname{tr}(K), \\
& h\left(k^{2}\right)=\operatorname{det}(D) k^{4}+\Gamma q k^{2}+\Gamma^{2} \operatorname{det}(K),
\end{aligned}
$$

with:

$$
\begin{aligned}
& K=\left(\begin{array}{cc}
b-1 & Q^{2} \\
-\frac{b}{\eta^{2}} & -\left(\frac{Q}{\eta}\right)^{2}
\end{array}\right) \\
& D=\left(\begin{array}{cc}
(m+1) Q^{m} & 0 \\
0 & \frac{n+1}{\eta^{2}}\left(\frac{b}{Q}\right)^{n}
\end{array}\right),
\end{aligned}
$$

and $q=-K_{11} D_{22}-K_{22} D_{11}$. The steady state $(\bar{u}, \bar{v})$ can lose its stability both via Hopf and Turing bifurcation. Oscillatory instability occurs when $g\left(k^{2}\right)=0$ and $h\left(k^{2}\right)>0$. The minimum values of $b$ and $k$ for which $g\left(k^{2}\right)=0$ are:

$$
b_{c}^{H}=1+\frac{Q^{2}}{\eta^{2}} \quad k=0,
$$

and for $b>b_{c}^{H}$ a spatially homogeneous oscillatory mode emerges. The neutral stability Turing boundary corresponds to $h\left(k^{2}\right)=0$, which has a single minimum $\left(k_{c}^{2}, b^{c}\right)$ attained when:

$$
k_{c}^{2}=-\Gamma \frac{(1-b)(n+1)\left(\frac{b}{Q}\right)^{n}+(m+1) Q^{m+2}}{2(m+1)(n+1) Q^{m-n} b^{n}},
$$

which requires $q<0$, therefore $b>1$ is a necessary condition for Turing instability. Then, the Turing bifurcation value $b=b^{c}$ is obtained by imposing $q^{2}-4 \operatorname{det}(D) \operatorname{det}(K)=0$ (under the condition $q<0$ ), which leads to solve:

$$
\left\{\begin{aligned}
((n+1) & \left.\left(\frac{b}{Q}\right)^{n}(1-b)+(m+1) Q^{m+2}\right)^{2} \\
& -4(m+1)(n+1) Q^{m-n+2} b^{n}=0 \\
Q^{m+n+2} & <\frac{(n+1)}{(m+1)} b^{n}(b-1) .
\end{aligned}\right.
$$


From (2.7) and (2.8) one can easily see that the first mode to lose stability, that we shall denote with $k_{c}$, and the bifurcation value $b^{c}$ do not depend explicitly on $\eta$. However being impossible to give an explicit expression, we have evaluated $k_{c}$ and $b^{c}$ numerically.

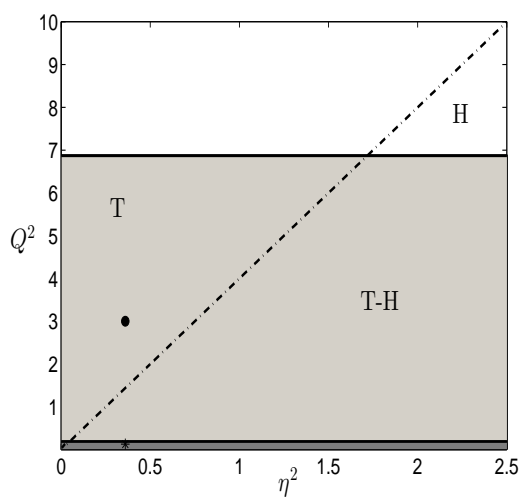

Figure 1: Hopf (dash-dotted line) and Turing (solid line) stability boundaries in the two dimensional slice $\{b=\mathrm{const}\}$, with $b>b_{c}$. The instability Turing region is shadowed in grey: the supercritical region in light grey, the subcritical region in dark grey (see Sections 3.1 and 3.2 below). The pure Hopf region, the pure Turing region and the region in which both Turing and Hopf instability occur are labeled with $\mathrm{H}, \mathrm{T}$ and $\mathrm{T}-\mathrm{H}$ respectively. The parameters are chosen as $m=n=1$ and $b=11>b_{c}=10.74$.

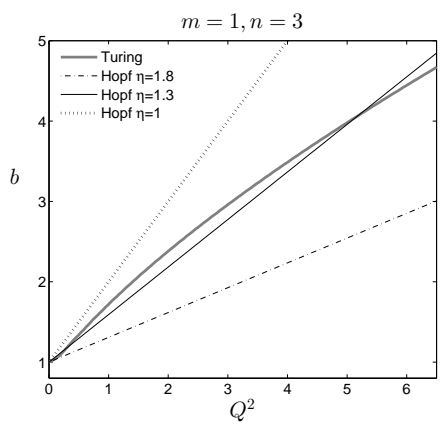

(a)

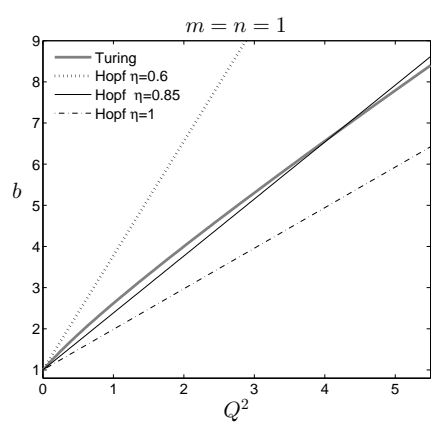

(b)

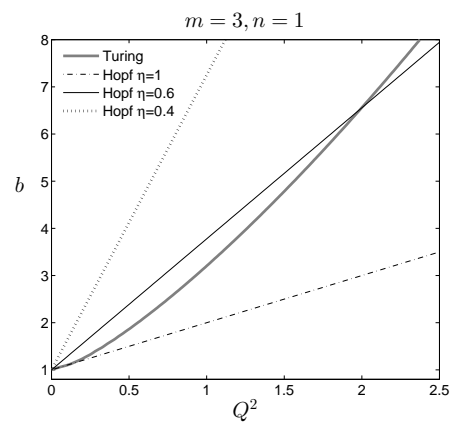

(c)

Figure 2: In Figs (a), (b), (c) we represent the Hopf and Turing stability boundaries for different values of $\eta$. The instability regions stay above the lines.

In Fig 1 we show the Turing and Hopf instability regions in the parameter space $\left(\eta^{2}, Q^{2}\right)$ for a fixed value $b>b^{c}$. In the region marked with TH there is a competition between the two instabilities; which one, between the Turing and the Hopf instability develops, depends on the locations of the respective instability boundaries: as $b$ increases, if $b_{c}^{H}>b^{c}$, Turing instability occurs prior to the oscillatory instability.

In Fig 2(a), (b) and (c) we report the Hopf and Turing instability boundaries for different values of $\eta$ and different values of the coefficients $m$ and $n$ expressing the nonlinearity of the 
diffusion. We observe that, while the Turing instability region does not depend on $\eta$, the Turing pattern region decreases by increasing $\eta$ due to the fact that $b_{c}^{H}$ decreases with $\eta$. Comparing the three Figs 2(a)-2(c) it is interesting to notice that, taking larger values of $m$ with respect to $n$, the shape of the Turing boundaries changes concavity; which leads to the fact that Turing patterns develop for large enough values of $Q$ when $m$ is smaller than $n$ (see Fig!2(a)), and for small enough values of $Q$ when $m$ is larger than $n$ (see Fig.2(c)) .

The effect of nonlinear diffusion is to make easier the formation of Turing pattern with respect to the case of classical diffusion (i.e. when $m=n=0$ ). This is apparent from Fig 3 where we have reported the stability boundaries for different values of $m$ and $n$ and keeping $\eta=1$.

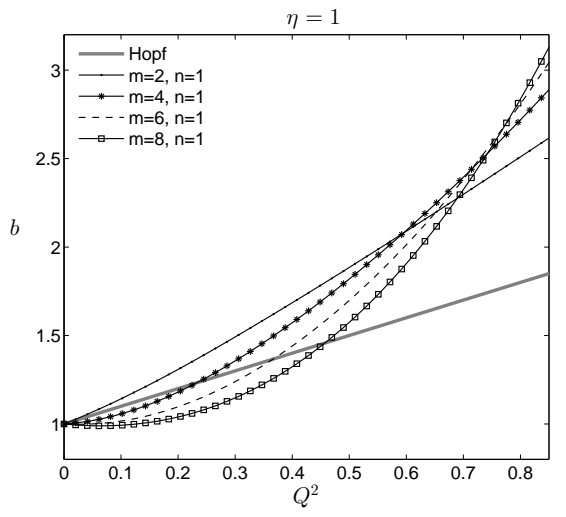

(a)

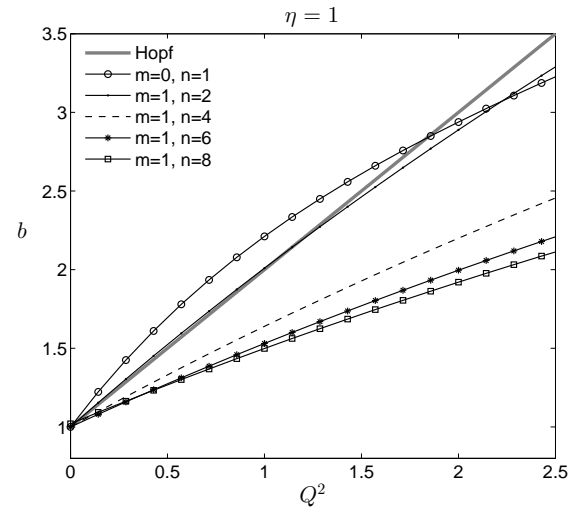

(b)

Figure 3: Hopf and Turing stability boundaries varying $m$ and $n$. The instability regions stay above the lines.

\section{$3 \quad$ Weakly nonlinear analysis}

We introduce the control parameter $\varepsilon$, representing the dimensionless distance from the threshold and defined as $\varepsilon^{2}=\left(b-b^{c}\right) / b^{c}$; then we recast the original system (1.1) in the following form:

$$
\partial_{t} \mathbf{w}=\mathcal{L}^{b} \mathbf{w}+\mathcal{N} \mathcal{L}^{b} \mathbf{w}, \quad \mathbf{w} \equiv\left(\begin{array}{c}
u-\bar{u} \\
v-\bar{v}
\end{array}\right),
$$

where the linear operator $\mathcal{L}^{b}=\Gamma K^{b}+D^{b} \nabla^{2}$ results from the linearization of the kinetics and of the diffusion term around the steady state $(\bar{u}, \bar{v})$, the matrix $K^{b}$ and $D^{b}$ being given in (2.5); here the dependence on the bifurcation parameter $b$ is made explicit for notational convenience. The nonlinear operator $\mathcal{N} \mathcal{L}^{b}$ represents the nonlinear remaining terms. Moreover we expand $\mathbf{w}$, the time $t$ and the bifurcation parameter $b$ as:

$$
\begin{aligned}
\mathbf{w} & =\varepsilon \mathbf{w}_{1}+\varepsilon^{2} \mathbf{w}_{2}+\ldots \quad t=t+\varepsilon T_{1}+\varepsilon^{2} T_{2}+\ldots \\
b & =b^{c}+\varepsilon b^{(1)}+\varepsilon^{2} b^{(2)}+\ldots
\end{aligned}
$$

Substituting the above expansions into (3.1) and collecting the terms at each order in $\varepsilon$, we obtain the following sequence of equations for the $\mathbf{w}_{i}$ : 
$O(\varepsilon):$

$$
\mathcal{L}^{b^{c}} \mathbf{w}_{1}=\mathbf{0}
$$

$O\left(\varepsilon^{2}\right):$

$$
\mathcal{L}^{b^{c}} \mathbf{w}_{2}=\mathbf{F}
$$

$O\left(\varepsilon^{3}\right):$

$$
\mathcal{L}^{b^{c}} \mathbf{w}_{3}=\mathbf{G}
$$

where:

$$
\begin{aligned}
& \mathbf{F}=\frac{\partial \mathbf{w}_{1}}{\partial T_{1}}-D^{(1)} \nabla^{2}\left(\left(v_{1}+\frac{b_{1}^{2}}{Q}\right)^{(1)}\right)+\left(\begin{array}{cc}
-b^{(1)} & 0 \\
b^{(1)} & 0
\end{array}\right) \mathbf{w}_{1} \\
& +\left(2 Q u_{1} v_{1}+\frac{b^{c}}{Q} u_{1}^{2}\right) \underline{\underline{1}}, \\
& \mathbf{G}=\frac{\partial \mathbf{w}_{1}}{\partial T_{2}}+\frac{\partial \mathbf{w}_{2}}{\partial T_{1}}-D^{(2)} \nabla^{2}\left(\left(v_{1}+\frac{b^{(1)}}{Q}\right)^{3}\right) \\
& -2 D^{(1)} \nabla^{2}\left(\left(v_{1}+\frac{b^{(1)}}{Q}\right)\left(v_{2}+\frac{b^{(2)}}{Q}\right)\right) \\
& +2\left(Q\left(u_{1} v_{2}+u_{2} v_{1}\right)+\frac{b^{c} u_{1} u_{2}}{Q}+\frac{u_{1}^{2}}{2}\left(v_{1}+\frac{b^{(1)}}{Q}\right)\right) \underline{\underline{ }} \\
& +\left(\begin{array}{cc}
-b^{(1)} & 0 \\
b^{(1)} & 0
\end{array}\right) \mathbf{w}_{2}+\left(\begin{array}{cc}
-b^{(2)} & 0 \\
b^{(2)} & 0
\end{array}\right) \mathbf{w}_{1},
\end{aligned}
$$

and:

$$
\begin{gathered}
\underline{\mathbf{1}}=\left(\begin{array}{c}
-1 \\
1
\end{array}\right), \\
D^{(1)}=\left(\begin{array}{cc}
\frac{m(m+1)}{2} Q^{m-1} & 0 \\
0 & \frac{n(n+1)}{2 \eta^{2}}\left(\frac{b^{c}}{Q}\right)^{n-1}
\end{array}\right), \\
D^{(2)}=\left(\begin{array}{cc}
\frac{m\left(m^{2}-1\right)}{6} Q^{m-2} & 0 \\
0 & \frac{n\left(n^{2}-1\right)}{6 \eta^{2}}\left(\frac{b^{c}}{Q}\right)^{n-2}
\end{array}\right) .
\end{gathered}
$$

The solution to the linear problem (3.3), satisfying the Neumann boundary conditions, is given by:

$$
\mathbf{w}_{1}=A\left(T_{1}, T_{2}\right) \boldsymbol{\rho} \cos \left(\bar{k}_{c} x\right),
$$

with $\boldsymbol{\rho} \in \operatorname{Ker}\left(\Gamma K^{b^{c}}-\bar{k}_{c}^{2} D^{b^{c}}\right)$ and $\bar{k}_{c}$ is the first admissible unstable mode. Once substituted the solution (3.6) in (3.4), the vector $\mathbf{F}$ is made orthogonal to the kernel of the adjoint of 


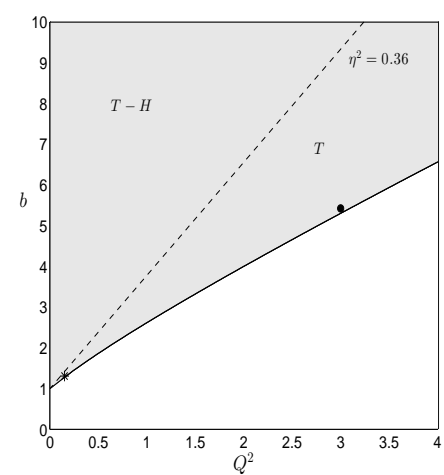

(a)

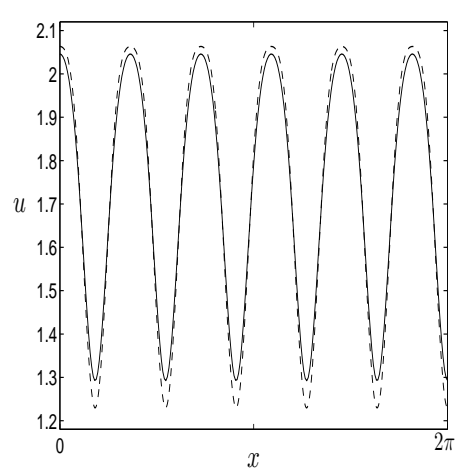

(b)

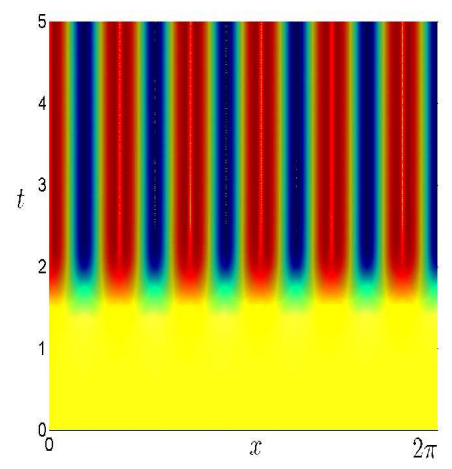

(c)

Figure 4: (a) The Turing instability (solid line) occurs prior to oscillatory instability (dashed line). (b) Comparison between the weakly nonlinear solution (dotted line) and the numerical solution of (1.1) (solid line). (c) Pattern evolution in the space-time plane. The parameters are chosen in the supercritical region $\Gamma=80, m=n=1, Q^{2}=3, \eta^{2}=0.36, \varepsilon=0.1$, corresponding to the point marked with the dark circle both in Fig 1 and in Fig 4(a). With this choice of the parameters one has $b^{c} \approx 5.3028$, while $\bar{k}_{c}=5.5$.

$\mathcal{L}^{b^{c}}$ simply by imposing $T_{1}=0$ and $b^{(1)}=0$. The solution of (3.4) can therefore be obtained right away and substituted into the linear problem (3.5) at order $\varepsilon^{3}$. The vector $\mathbf{G}$ has the following expression:

$$
\mathbf{G}=\left(\frac{d A}{d T} \boldsymbol{\rho}+A \mathbf{G}_{1}^{(1)}+A^{3} \mathbf{G}_{1}^{(3)}\right) \cos \left(\bar{k}_{c} x\right)+\mathbf{G}^{*},
$$

where $T=T_{2}, \mathbf{G}_{1}^{j}, j=1,3$ and $\mathbf{G}^{*}$ (which contains automatically orthogonal terms) depend on the parameters of the original system (1.1) and their explicit expression is here omitted as it is too cumbersome. The elimination of secular terms in the equation (3.5) results in the following Stuart-Landau equation for the amplitude $A(T)$ :

$$
\frac{d A}{d T}=\sigma A-L A^{3}
$$

where $\sigma$ and $L$ are explicitly computed in terms of the system parameters:

$$
\sigma=-\frac{<\mathbf{G}_{1}^{(1)}, \boldsymbol{\psi}>}{<\boldsymbol{\rho}, \boldsymbol{\psi}>}, \quad L=\frac{<\mathbf{G}_{1}^{(3)}, \boldsymbol{\psi}>}{<\boldsymbol{\rho}, \boldsymbol{\psi}>}
$$

and $\psi \in \operatorname{Ker}\left\{\left(\Gamma K^{b^{c}}-\bar{k}_{c}^{2} D^{b^{c}}\right)^{\dagger}\right\}$. Experimental evidence shows that, when the domain size is large, the pattern sequentially forms and travelling wavefronts are the precursors to patterning. Therefore the amplitude of the pattern is also modulated in space. The slow spatial scale $X=\varepsilon x$ can be easily obtained from the linear analysis. At $O(\varepsilon)$ we still recover the linear problem $\mathcal{L}^{b^{c}} \mathbf{w}_{1}=\mathbf{0}$ and the solution is as in (3.1), but $A \equiv A(X, T)$ also depends on the slow spatial scale $X$. Dealing with the equations at $O\left(\varepsilon^{2}\right)$ and $O\left(\varepsilon^{3}\right)$ as before, we 

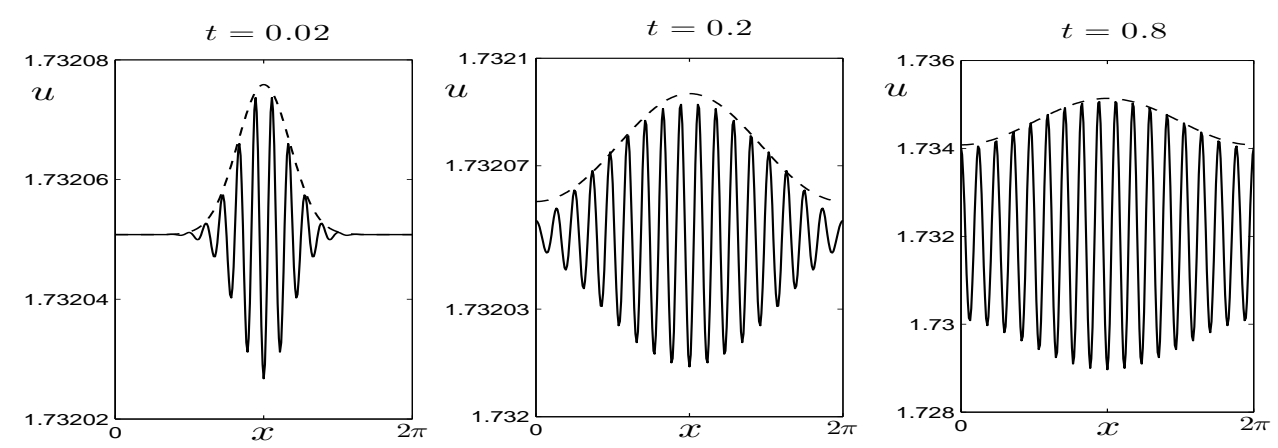

Figure 5: The equilibrium solution is perturbed at the center of the spatial interval. The pattern forms as a modulated wave (solid line). The dashed line is a numerical solution of the Ginzburg-Landau equation (3.10). The parameters are chosen as in Fig 4, with $\Gamma=800$. Here $\varepsilon=10^{-3}$.

obtain the following Ginzburg-Landau equation for the amplitude $A(X, T)$ :

$$
\frac{\partial A}{\partial T}=\nu \frac{\partial^{2} A}{\partial X^{2}}+\sigma A-L A^{3}
$$

where:

$$
\nu=-\frac{<2 \bar{k}_{c} D^{b^{c}} \mathbf{w}_{21}+D^{b^{c}} \boldsymbol{\rho} . \boldsymbol{\psi}>}{<\boldsymbol{\rho}, \boldsymbol{\psi}>} .
$$

Here $\mathbf{w}_{21}$ is the solution of the following linear system:

$$
\left(\Gamma K^{b^{c}}-\bar{k}_{c}^{2} D^{b^{c}}\right) \mathbf{w}_{21}=-2 \bar{k}_{c} D^{b^{c}} \boldsymbol{\rho}
$$

and $\sigma$ and $L$ are the same as in formulas (3.9).

\subsection{The supercritical case}

In the pattern-forming region, the growth rate coefficient $\sigma$ is always positive. Therefore two different qualitative dynamics of the Stuart-Landau equation (3.8) can be identified based on the sign of the coefficient $L: L>0$ corresponds to the supercritical case and $L<0$ to the subcritical case (see Fig 1). In the supercritical case the Stuart-Landau equation admits the stable equilibrium solution $A_{\infty}=\sqrt{\sigma / L}$, which corresponds to the asymptotic value of the amplitude $A$ of the pattern. In Fig 4 (b), we show the comparison between the stationary solution predicted by the weakly nonlinear analysis up to $O\left(\varepsilon^{2}\right)$ and the pattern solution computed solving numerically the system (1.1) starting from a random periodic perturbation of the constant state. In all the tests we have performed we have verified that the distance, evaluated in the $L^{1}$ norm, between the weakly nonlinear approximation and the numerical solution of the system is $O\left(\varepsilon^{3}\right)$. Let us consider the same parameter set as in Fig 4, except $\Gamma=800$ larger by a factor 10, which is equivalent to have a spatial domain larger by a factor $\sqrt{10}$. Once one perturbs the equilibrium solution at the center of the spatial domain, the pattern propagating as a wave is observed. In Fig 5 it is shown how the Ginzburg-Landau 


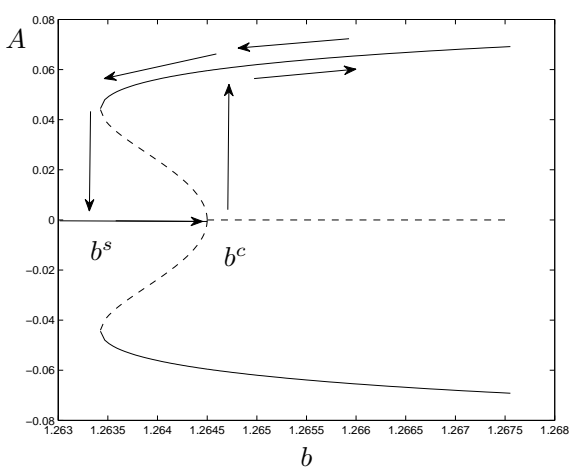

(a)

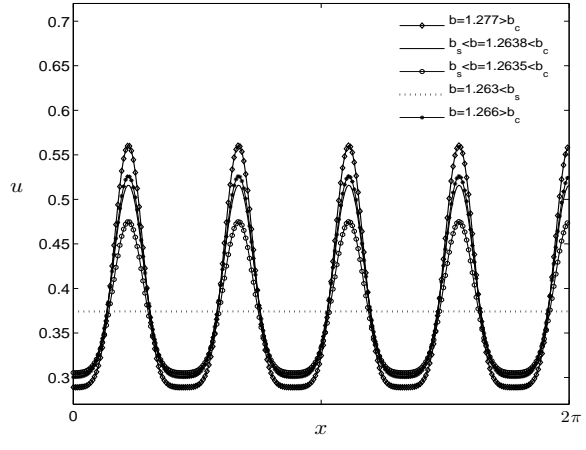

(b)

Figure 6: (a) The bifurcation diagram in the subcritical case. (b) A hysteresis cycle and the corresponding pattern evolution in the subcritical case. The bifurcation value $b$ varies following the order given into the legend. The parameters are $\Gamma=150, Q^{2}=0.14, \eta^{2}=0.36$, chosen in the subcritical region, at the point marked with an asterisk both in Fig 1 and in Fig斗(a). In this case $b^{c} \approx 1.2645, b^{s}=1.2634$.

equation (3.10) is able to capture the envelope evolution and the progressing of the pattern as a wave.

\subsection{The subcritical case}

In the subcritical region shadowed in dark grey in Fig,1 the Stuart-Landau equation (3.8) does not admit any stable equilibrium and it is not able to capture the amplitude of the pattern. We therefore need to push the weakly nonlinear analysis at a higher order (see [2] and references therein). The compatibility condition imposed at $O\left(\varepsilon^{5}\right)$ leads to the following quintic Stuart-Landau equation for the amplitude $A$ :

$$
\frac{d A}{d T}=\bar{\sigma} A-\bar{L} A^{3}+\bar{R} A^{5}
$$

where the coefficients $\bar{\sigma}, \bar{L}$ and $\bar{R}$ appearing in (3.13) are obtained in terms of the parameters of the original system (1.1). The explicit expressions are too involved and are not reported here; however we notice that $\bar{\sigma}$ and $\bar{L}$ are $O\left(\varepsilon^{2}\right)$ perturbation of the coefficients of the cubic Stuart-Landau equation, while $\bar{R}=O\left(\varepsilon^{2}\right)$; this leads to $A=O\left(\varepsilon^{-1}\right)$ consistently with the reported bifurcation diagram. When $\bar{\sigma}>0, \bar{L}<0$ and $\bar{R}<0$, the equation (3.13) admits two symmetric real stable equilibria, corresponding to the asymptotic values of the amplitude $A$.

On the left of Fig 6, the bifurcation diagram is shown for the values of the parameters chosen in the subcritical region. When $b^{s}<b<b^{c}$ both the origin and two large amplitude branches are stable, indicating the possibility of hysteresis as $b$ is varied. On the right of Fig [6, it is shown how the pattern forms starting with a value of $b>b^{c}$, as the solution of the equation (3.13) jumps to the large amplitude stable branch. Moreover, decreasing $b$, with $b^{s}<b<b^{c}$ this pattern solution persists; it disappears, reaching the constant steady state, only with a further decrease of $b$ below $b^{s}$, as the solution of (3.13) jumps to the origin. The pattern forms 
again by increasing the parameter $b$ above $b^{c}$. Finally, we notice that in the subcritical case the amplitude of the pattern is relatively insensitive to the size of the bifurcation parameter.

\section{Two dimensional domain}

In this section we shall investigate the pattern appearance for the reaction-diffusion system (1.1) in a two-dimensional domain (here $\zeta \in \Omega \subseteq \mathbb{R}^{2}$ ). Notice that the critical value for the bifurcation parameter and the critical wavenumber do not depend on the geometry of the domain and they are still computed via linear stability analysis as in Section 2 .

\subsection{Rectangular domain}

Let $\zeta \equiv(x, y) \in \Omega$, with $\Omega=\left[0, L_{x}\right] \times\left[0, L_{y}\right]$. The solutions to the linearized system associated to (1.1) with Neumann boundary conditions are:

$$
\begin{aligned}
& \mathbf{w}=\sum_{p, q \in \mathbb{N}} \mathbf{f}_{p q} e^{\sigma\left(k_{p q}^{2}\right) t} \cos (\phi x) \cos (\psi y), \\
& k_{p q}^{2}=\phi^{2}+\psi^{2}, \text { where } \phi \equiv \frac{p \pi}{L_{x}}, \quad \psi \equiv \frac{q \pi}{L_{y}},
\end{aligned}
$$

where $\mathbf{f}_{p q}$ are the Fourier coefficients of the initial conditions and $\sigma\left(k_{p q}^{2}\right)$ are computed via the dispersion relation (2.4). The range of the unstable wavenumbers of allowable patterns strictly depends on the domain geometry and the boundary conditions. Being the domain finite, to see a pattern emerging as $t$ increases, there should exist at least a mode pair $(p, q)$ such that:

$$
\begin{aligned}
& k_{1}^{2}<k^{2} \equiv \phi^{2}+\psi^{2}<k_{2}^{2} \text { and } \sigma\left(k^{2}\right)>0 \\
& \text { where } \phi \equiv \frac{p \pi}{L_{x}} \text { and } \psi \equiv \frac{q \pi}{L_{y}}
\end{aligned}
$$

i.e. for $b>b^{c}$ and $\Gamma$ sufficiently large (as the unstable wavenumbers $k_{1}^{2}$ and $k_{2}^{2}$ are proportional to $\Gamma$ ). Our analysis will be restricted to the case when only one admissible unstable eigenvalue, here denoted with $\bar{k}_{c}$, falls within the band $\left(k_{1}, k_{2}\right)$. Given $\bar{k}_{c} \in\left[k_{1}, k_{2}\right]$, the degeneracy phenomenon can occur: one, two or more pairs $(p, q)$ may exist such that $\bar{k}_{c}^{2}=\phi^{2}+\psi^{2}$ and the corresponding eigenvalue $\sigma$ will have single, double or higher multiplicity giving rise to different types of linear patterns.

The weakly nonlinear analysis can be once again carried out to obtain the equations which rule the evolution of the pattern amplitude near the threshold. The solution of the linear problem as in (3.3), satisfying the Neumann boundary conditions, is given by:

$$
\mathbf{w}_{1}=\sum_{i=1}^{r} A_{i}\left(T_{1}, T_{2}\right) \boldsymbol{\rho} \cos \left(\phi_{i} x\right) \cos \left(\psi_{i} y\right),
$$

where $r$ is the multiplicity of the eigenvalue, $A_{i}$ are the slowly varying amplitudes (still arbitrary at this level) and $\rho \in \operatorname{Ker}\left(\Gamma K^{b^{c}}-\bar{k}_{c}^{2} D^{b^{c}}\right)$. We shall show the types of supported patterns when the multiplicity is $r=1$ or $r=2$. 


\subsubsection{Simple eigenvalue $r=1$}

When $r=1$ the multiple scales method strictly follows the analysis given in Section 3, The amplitude equation, still recovered at $O\left(\varepsilon^{3}\right)$, is the Stuart-Landau equation (3.8) and the emerging solution of the reaction-diffusion system (1.1) in the supercritical case is given by:

$$
\mathbf{w}=\varepsilon \boldsymbol{\rho} A_{\infty} \cos (\phi x) \cos (\psi y)+O\left(\varepsilon^{2}\right),
$$

where $A_{\infty}$ is the stable stationary state of the Stuart-Landau equation (3.8). These solutions are rhombic spatial patterns (see [5]), whose special cases are the rolls (when $\phi$ or $\psi$ is zero) or the squares (when $\phi=\psi$ ). The numerical solution, obtained via spectral methods, of the system (1.1), starting from an initial datum which is a random periodic perturbation about the steady state $(\bar{u}, \bar{v})$, stabilizes to the roll pattern shown in Fig 7. The system parameters are chosen in such a way that, in the rectangular domain $L_{x}=\pi$ and $L_{y}=\sqrt{3} \pi$, only the most unstable mode $\bar{k}_{c}^{2}=3$ satisfies the condition (4.3) and the corresponding eigenvalue is single, as the uniform steady state is linearly unstable to the unique mode pair $(p, q)=(0,3)$. For a better presentation of the results the amplitude of the zero mode (corresponding to the equilibrium solution) has been set equal to zero into the figures representing the spectrum of the solution. We have verified that the error in predicting the amplitude of the pattern using (4.5) is $O\left(\varepsilon^{2}\right)$ (see the presence of the subharmonic $(0,6)$ in Fig 7 (b) which can be estimated including into the approximated solution (4.5) also the terms at $O\left(\varepsilon^{2}\right)$ ).

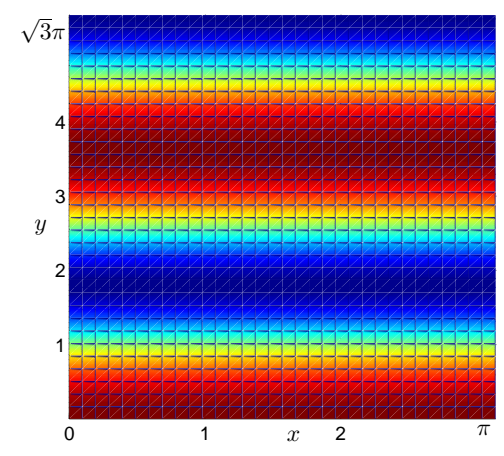

(a)

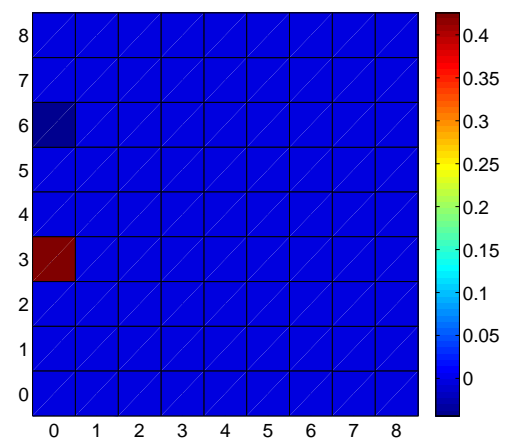

(b)

Figure 7: (a) The species $u$. (b) Spectrum of the numerical solution. The parameters are $\Gamma=8, m=n=1, Q^{2}=3, \eta^{2}=0.36, b=b^{c}\left(1+\varepsilon^{2}\right)$, where $b^{c}=5.3028$ and $\varepsilon=0.05$.

In the square domain with $L_{x}=L_{y}=\pi$, we have picked the parameters values as in the caption of Fig 8, such that the conditions in (4.3) are satisfied by the unique discrete unstable mode $\bar{k}_{c}^{2}=8$ and the mode pair $(p, q)=(2,2)$. From an initial condition which is a random periodic perturbation about $(\bar{u}, \bar{v})$, the numerical solution of the system (1.1) stabilizes to the square pattern given in Fig 8 , in agreement with the expected solution (4.5). The subharmonics $(4,0),(0,4)$ and $(4,4)$ in Fig $[8(\mathrm{~b})$ can be predicted via the weakly nonlinear approximation (4.5) up to $O\left(\varepsilon^{2}\right)$. 


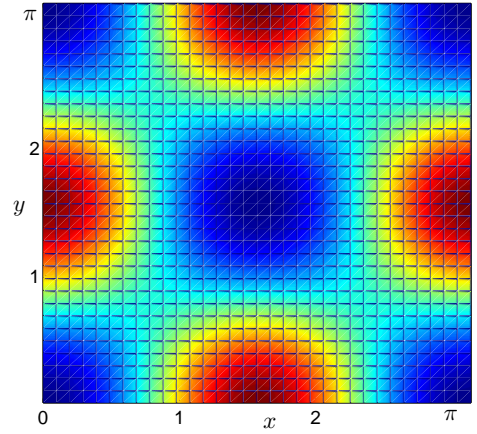

(a)

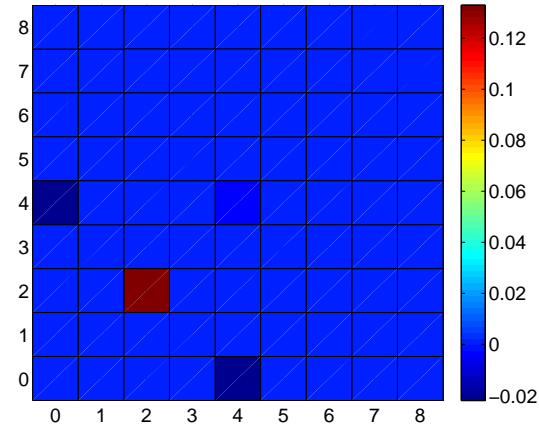

(b)

Figure 8: (a) The species $u$. (b) Spectrum of the numerical solution. The parameters are $\Gamma=30.3, m=1, n=2, Q^{2}=3.5, \eta^{2}=0.81, b=b^{c}\left(1+\varepsilon^{2}\right)$, where $b^{c}=3.9542$ and $\varepsilon=0.02$.

\subsubsection{Double eigenvalue $r=2$, no-resonance condition holds}

Let us assume that the multiplicity of the eigenvalue is $r=2$ and the following no-resonance condition holds:

$$
\begin{array}{lcc}
\phi_{i}+\phi_{j} \neq \phi_{j} & \text { or } & \psi_{i}-\psi_{j} \neq \psi_{j} \\
& \text { and } & \\
\phi_{i}-\phi_{j} \neq \phi_{j} & \text { or } & \psi_{i}+\psi_{j} \neq \psi_{j}
\end{array}
$$

with $i, j=1,2$ and $i \neq j$. Also in this case the weakly nonlinear analysis is performed up to $O\left(\varepsilon^{3}\right)$ and the solvability condition for the equation (3.5) leads to the following two coupled Landau equations for the amplitudes $A_{1}$ and $A_{2}$ :

$$
\begin{aligned}
& \frac{d A_{1}}{d T_{2}}=\sigma A_{1}-L_{1} A_{1}^{3}+R_{1} A_{1} A_{2}^{2} \\
& \frac{d A_{2}}{d T_{2}}=\sigma A_{2}-L_{2} A_{2}^{3}+R_{2} A_{1}^{2} A_{2} .
\end{aligned}
$$

In the supercritical case, when the system (4.7) admits at least one stable equilibrium $\left(A_{1 \infty}, A_{2 \infty}\right)$, the emerging asymptotic solution of the reaction-diffusion system (1.1) at the leading order is approximated by:

$$
\mathbf{w}=\varepsilon \boldsymbol{\rho} \sum_{i=1}^{2} A_{i \infty} \cos \left(\phi_{i} x\right) \cos \left(\psi_{i} y\right)+O\left(\varepsilon^{2}\right) .
$$

When $A_{1 \infty}$ or $A_{2 \infty}$ is zero, the solutions in (4.8) are the rhombic spatial patterns described in Section 4.1.1. When both $A_{i \infty} \neq 0, \quad i=1,2$, more complex structures arise due to the interaction of different modes $\phi_{i}, \psi_{i}$, the so-called mixed-mode patterns. The complete classification of the equilibrium points of the system (4.7) via linear stability analysis is given in [11. 


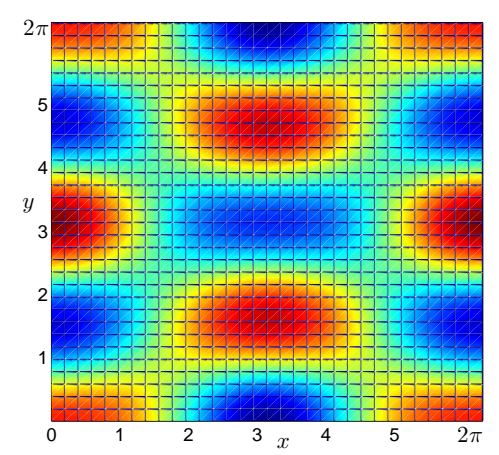

(a)

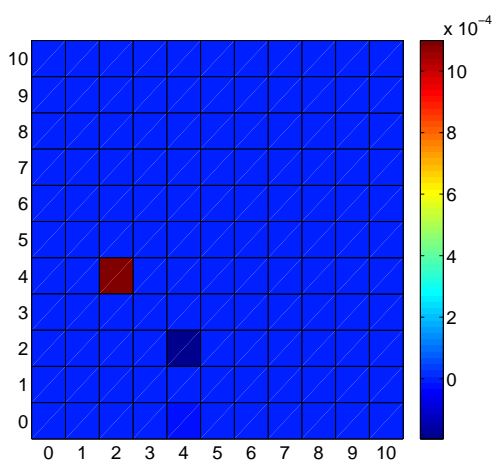

(b)

Figure 9: (a) The species $u$. (b) Spectrum of the numerical solution. The parameters are $\Gamma=11.93, m=n=1, Q^{2}=8, \eta^{2}=0.36, b=b^{c}\left(1+\varepsilon^{2}\right)$, where $b^{c}=11.3722$ and $\varepsilon=0.03$.

Let us consider a domain with dimensions $L_{x}=L_{y}=2 \pi$ and choose the parameter values as in the caption of Fig 9 in such a way that only the most unstable discrete mode $\bar{k}_{c}^{2}=5$ falls within the band of unstable modes allowed by the boundary conditions. In this case the eigenvalue is double, as the uniform steady state is linearly unstable to the two mode pairs $(2,4)$ and $(4,2)$. With this choice of the parameters the system (4.7) admits only one stable equilibrium $\left(A_{1 \infty}, A_{2 \infty}\right)$ with both nonzero coordinates and the expected solution in (4.8) agrees with the numerical asymptotic solution of the system (1.1), having as initial datum a random periodic perturbation about the steady state $(\bar{u}, \bar{v})$, shown in Fig.(9).

\subsubsection{Double eigenvalue $r=2$, resonance condition holds}

Let the multiplicity of the eigenvalue be $r=2$ and the resonance condition be satisfied as follows:

$$
\begin{aligned}
& \phi_{i}+\phi_{j}=\phi_{j} \quad \text { and } \quad \psi_{i}-\psi_{j}=\psi_{j} \\
& \text { or } \\
& \phi_{i}-\phi_{j}=\phi_{j} \quad \text { and } \quad \psi_{i}+\psi_{j}=\psi_{j}
\end{aligned}
$$

with $i, j=1,2$ and $i \neq j$. Assuming, without loss of generality, that the second condition in (4.9) holds with $i=2$ and $j=1$, and taking into account the relation in (4.3), it follows that $\phi_{2}=2 \phi_{1}, \psi_{2}=0, \psi_{1}=\sqrt{3} \phi_{1}, \phi_{1}=\bar{k}_{c} / 2$ and $L_{y}=\sqrt{3} L_{x}$. In this case the secular terms appear at $O\left(\varepsilon^{2}\right)$ in (3.4); however the amplitude equations one derives imposing the solvability condition do not admit stable equilibrium in any parameter regimes. Therefore the asymptotic analysis has to be pushed to higher order, see [2, 11]. By the Fredholm alternative for the equation (3.5), at $O\left(\varepsilon^{3}\right)$ one finds the following system for the amplitudes $A_{1}$ and $A_{2}$ :

$$
\begin{aligned}
& \frac{d A_{1}}{d T}=\sigma_{1} A_{1}-L_{1} A_{1} A_{2}+R_{1} A_{1} A_{2}^{2}+S_{1} A_{1}^{3}, \\
& \frac{d A_{2}}{d T}=\sigma_{2} A_{2}-L_{2} A_{1}^{2}+R_{2} A_{1}^{2} A_{2}+S_{2} A_{2}^{3},
\end{aligned}
$$




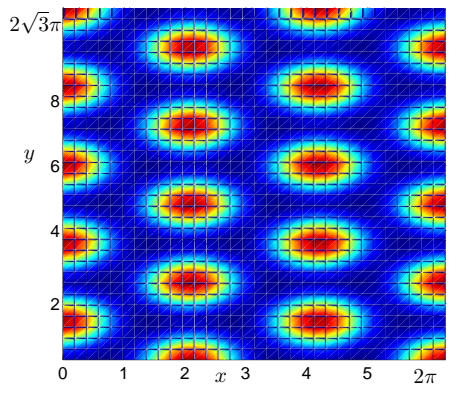

(a)

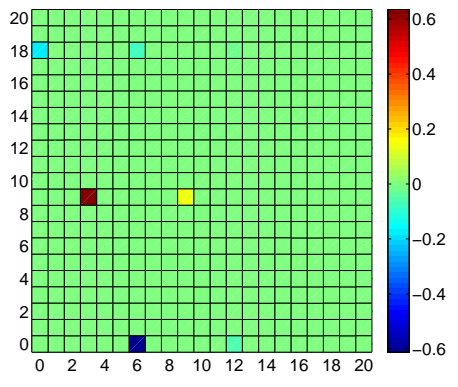

(b)

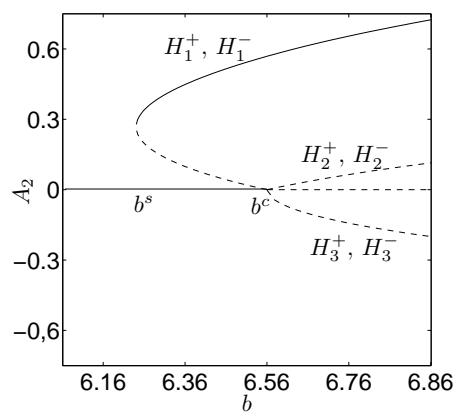

(c)

Figure 10: (a) The species $u$. (b) Spectrum of the numerical solution. (c) The bifurcation diagram. The parameters are $\Gamma=23.054, m=n=1, Q^{2}=4, \eta^{2}=0.3025, b=b^{c}\left(1+\varepsilon^{2}\right)$, where $b^{c}=6.5615$ and $\varepsilon=0.01$.

where $\sigma_{i}$ and $L_{i}$ are $O\left(\varepsilon^{2}\right)$ perturbation of the coefficients of the amplitude equations found at $O\left(\varepsilon^{2}\right)$, while $R_{i}$ and $S_{i}$ are $O\left(\varepsilon^{2}\right)$. At the leading order, the emerging asymptotic solution of the system (1.1) is approximated by:

$$
\begin{aligned}
\mathbf{w}=\varepsilon \boldsymbol{\rho}( & A_{1 \infty} \cos \left(\phi_{1} x\right) \cos \left(\psi_{1} y\right) \\
& \left.+A_{2 \infty} \cos \left(\phi_{2} x\right) \cos \left(\psi_{2} y\right)\right)+O\left(\varepsilon^{2}\right),
\end{aligned}
$$

where $\left(A_{1 \infty}, A_{2 \infty}\right)$ is a stable state of the system (4.10). The possible stationary states of the system (4.10) are $R^{ \pm} \equiv\left(0, \pm \sqrt{-\sigma_{2} / S_{2}}\right)$ and the six roots $H_{i}^{ \pm} \equiv\left(A_{1 i}^{ \pm}, A_{2 i}\right), i=1,2,3$, of the following system:

$$
\left\{\begin{array}{c}
A_{2}^{3}\left(S_{1} S_{2}-R_{1} R_{2}\right)+A_{2}^{2}\left(L_{1} R_{2}+L_{2} R_{1}\right)+ \\
A_{2}\left(S_{1} \sigma_{2}-L_{1} L_{2}-R_{2} \sigma_{1}\right)+L_{2} \sigma_{1}=0, \\
A_{1}^{2}=\frac{1}{S_{1}}\left(-R_{1} A_{2}^{2}+L_{1} A_{2}-\sigma_{1}\right) .
\end{array}\right.
$$

When $R^{ \pm}$or $H_{i}^{ \pm}$exist real and stable, the corresponding solution (4.11) is respectively a roll or a hexagonal pattern. In Fig 10 we show the hexagonal pattern which forms starting from an initial datum which is a random periodic perturbation about the steady state $(\bar{u}, \bar{v})$. For the parameters chosen as in the caption of Fig 10, only the mode $\bar{k}_{c}^{2}=9$ is admitted by the boundary conditions in the rectangular domain with $L_{x}=2 \pi$ and $L_{y}=2 \sqrt{3} \pi$. The eigenvalue predicted by the linear analysis is double as both the two pairs $(3,9)$ and $(6,0)$ satisfy the conditions in (4.3). The weakly nonlinear analysis predicts that only the states $H_{1}^{ \pm}$are stable (as shown in the bifurcation diagram in Fig[10(c)). The form of the pattern emerging from a numerical simulation of the full system, see Fig[10(a), is qualitatively well captured by the hexagonal pattern (4.11) predicted by the WNL analysis, which however underestimates the sub-harmonics shown in Fig 10(b), as it is usual for subcritical cases.

\subsection{Target pattern with radial symmetry}

Giving a small radially symmetric perturbation of the uniform equilibrium at the center of a square domain, the emerging solution of the system (1.1) is the axisymmetric pattern shown 
in Fig[11] It is a typical target pattern showing a larger amplitude at the center, see Fig[11(b).

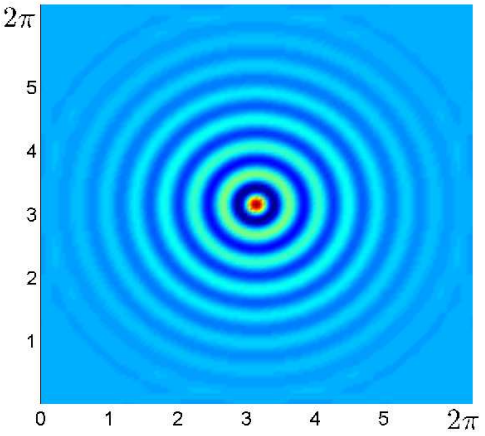

(a)

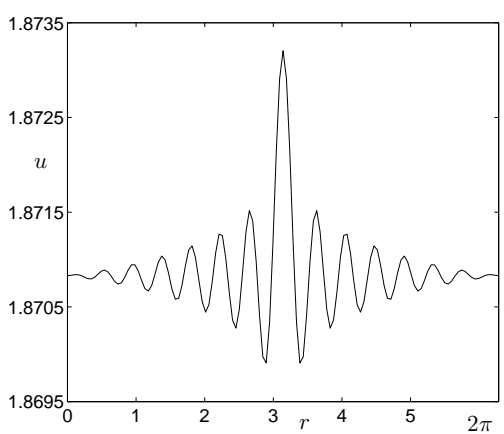

(b)

Figure 11: (Color online) (a) Target pattern. (b) The cross-section.

The weakly nonlinear analysis can be performed to recover the Ginzburg-Landau equation which captures the amplitude of the fluctuations of the target pattern close to the threshold [35, 40]. Let $r$ be the spatial radial coordinate, the amplitude of the pattern depends on the slow spatial scale $R=\varepsilon r$. Away from the core the curvature effects can be neglected and the following Ginzburg-Landau equation is easily derived following the procedure as in Section 3.

$$
\frac{\partial A}{\partial T}=\nu\left(\frac{\partial^{2} A}{\partial R^{2}}+\frac{1}{R} \frac{\partial A}{\partial R}-\frac{A}{4 R^{2}}\right)+\sigma A-L A^{3} .
$$

The envelope evolution of the outer solution $\mathbf{w}$ is therefore approximated by:

$$
\mathbf{w}_{O}=\varepsilon A(R, T) \mathbf{w}_{21} \cos \left(k_{c} \bar{r}\right)+O\left(\varepsilon^{2}\right),
$$

where $\bar{r}=r-\pi / 4$ and $\mathbf{w}_{21}$ is the solution of a linear system as in (3.12). Let us rewrite the amplitude equation (4.12) in terms of the variable $\mathcal{A}=A R^{1 / 2}$ as it simplifies to the following form:

$$
\frac{\partial \mathcal{A}}{\partial T}=\partial_{R R} \mathcal{A}+\sigma \mathcal{A}-L \frac{\mathcal{A}^{3}}{R} .
$$

The amplitude equation (4.14) does not hold close to the center of the target pattern where the curvature terms cannot be neglected. Taking into account the curvature terms, through a linear analysis as in 27] we get the following inner solution (close to the core of the pattern) depending on the spatial radial coordinate $r$ :

$$
\mathbf{w}_{I}=C \mathbf{w}_{21} J_{0}\left(k_{c} r\right),
$$

where $J_{0}$ is the zeroth order Bessel function of first kind and $C$ is a constant.

The solution of the equation (4.14) when $R \rightarrow 0$ should match with the solution (4.15) when $r \rightarrow \infty$. The behavior of the solution of the equation (4.14) when $R \rightarrow 0$ is the following:

$$
\mathcal{A} \approx a+b R+a|a|^{2} R \log R,
$$


where $a$ and $b$ are constants, see [33]. Using the well known asymptotic formula for the Bessel function $J_{0}$, one finds that, when $r \rightarrow \infty$, the inner solution behaves as:

$$
\mathbf{w}_{I} \approx \frac{C \mathbf{w}_{21}}{\sqrt{\pi k_{c} \bar{r}}} \cos \left(k_{c} \bar{r}\right) .
$$

The matching between the two solutions leads to the constant $C$ being $O\left(\varepsilon^{1 / 2}\right)$. Therefore the solution in the core $\mathbf{w}_{I}=\varepsilon^{1 / 2} \mathbf{w}_{21} J_{0}\left(k_{c} r\right)$ is larger than in the outer region [29]. This explains the larger amplitude at the center of the axisymmetric solution of the system (1.1) observed in Fig 11(b).

\section{Conclusions}

In this paper we have analyzed a two-species reaction-diffusion system which models the Brusselator dynamics with nonlinear density-dependent diffusion. We have firstly derived the conditions both for Turing and oscillatory instabilities, showing that the presence of nonlinear diffusion extends the range of diffusion coefficients over which Turing patterns can occur, in particular even when the diffusion coefficient of the activator exceeds that one of the inhibitor.

In one dimensional domain the supercritical or the subcritical character of the Turing bifurcation has been determined by deriving the amplitude equation for patterns near the instability threshold via weakly nonlinear analysis. In the subcritical case we have also shown that the system exhibits hysteresis, as the amplitude equation supports bistability. Moreover, when the domain is large, we have observed the pattern forming sequentially and invading the whole domain as a traveling wavefront, whose evolution is governed by the Ginzburg-Landau equation.

In a two dimensional rectangular domain we have observed a rich scenario of diverse patterns, such as rolls, squares and mixed-mode patterns emerging due to the interaction of different modes. Among mixed-mode patterns we have also shown the hexagonal patterns, arising when a resonance condition holds. We have employed the weakly nonlinear analysis to obtain the amplitude equations in each case and numerical simulations of the reaction-diffusion system exhibit the features predicted by these amplitude equations. Finally the analysis has been moved to target pattern with radial symmetry. Since this wave pattern shows a larger amplitude near the center of its circular profile than in its traveling fluctuations, we have applied a matching procedure to appropriately approximate the amplitude solution.

Some aspects of the problem remain to be examined. As the homogeneous state can lose its stability via a Hopf bifurcation, non-stationary patterns should also develop. The weakly nonlinear analysis can be employed to obtain the amplitude equations both near the Hopf bifurcation point [16] and next to the codimension-2 Turing-Hopf point here determined [38. This will be the subject of a forthcoming paper.

\section{Acknowledgements}

The work of the first (GG) and fourth (VS) authors has been partially supported by GNFM/INdAM through a Progetto Giovani grant. 


\section{References}

[1] Severine Atis, Sandeep Saha, Harold Auradou, Dominique Salin, and Laurent Talon. Autocatalytic Reaction Fronts Inside a Porous Medium of Glass Spheres. Phys. Rev. Lett., 110(14), APR 22013.

[2] P. Becherer, A. N. Morozov, and W. van Saarloos. Probing a subcritical instability with an amplitude expansion: An exploration of how far one can get. Physica D, 238(18):18271840, 2009.

[3] Benedetto Bozzini, Deborah Lacitignola, Claudio Mele, and Ivonne Sgura. Coupling of morphology and chemistry leads to morphogenesis in electrochemical metal growth: a review of the reaction-diffusion approach. Acta Appl. Math., 122:53-68, 2012.

[4] L. Chen and A. Jüngel. Analysis of a parabolic cross-diffusion semiconductor model with electron-hole scattering. Commun. Part. Diff. Eq., 32(1-3):127-148, 2007.

[5] G. C. Cruywagen, P. K. Maini, and J. D. Murray. Biological pattern formation on twodimensional spatial domains: a nonlinear bifurcation analysis. SIAM J. Appl. Math., 57(6):1485-1509, 1997.

[6] D. del Castillo-Negrete, B. A. Carreras, and V. Lynch. Front propagation and segregation in a reaction-diffusion model with cross-diffusion. Physica D, 168/169:45-60, 2002.

[7] J. M. Epstein and J. A. Pojman. An Introduction to Nonlinear Chemical Dynamics. Oxford University Press, Oxford, 1998.

[8] G. Galiano. On a cross-diffusion population model deduced from mutation and splitting of a single species. Comput. Math. Appl., 64(6):1927 - 1936, 2012.

[9] G. Gambino, M. C. Lombardo, and M. Sammartino. A velocity-diffusion method for a Lotka-Volterra system with nonlinear cross and self-diffusion. Appl. Numer. Math., 59(5):1059-1074, 2009.

[10] G. Gambino, M. C. Lombardo, and M. Sammartino. Turing instability and traveling fronts for a nonlinear reaction-diffusion system with cross-diffusion. Math. Comput. Simulat., 82(6):1112-1132, 2012.

[11] G. Gambino, M.C. Lombardo, and M. Sammartino. Pattern formation driven by crossdiffusion in a 2D domain. Nonlinear Anal. Real World Appl., 14(3):1755-1779, 2013.

[12] A. Golovin, B. Matkowsky, and V. Volpert. Turing pattern formation in the Brusselator model with superdiffusion. SIAM J. Appl. Math., 69(1):251-272, 2008.

[13] P. Gray and S. K. Scott. Chemical Oscillations and Instabilities. Oxford University Press, Oxford, 1997.

[14] M. Hildebrand. Self-organized nanostructures in surface chemical reactions: Mechanisms and mesoscopic modeling. Chaos, 12(1):144-156, 2002.

[15] R. Imbihl and G. Ertl. Oscillatory kinetics in heterogeneous catalysis. Chem. Rev., 95(3):697-733, 1995. 
[16] M. Ipsen, L. Kramer, and P.G. Sorensen. Amplitude equations for description of chemical reaction-diffusion systems. Phys. Rep., 337:193-235, 2000.

[17] A. Jüngel. Diffusive and nondiffusive population models. In Mathematical modeling of collective behavior in socio-economic and life sciences, Model. Simul. Sci. Eng. Technol., pages 397-425. Birkhäuser Boston Inc., Boston, MA, 2010.

[18] Niraj Kumar and Werner Horsthemke. Turing bifurcation in a reaction-diffusion system with density-dependent dispersal. Physica A, 389(9):1812 - 1818, 2010.

[19] Niraj Kumar and Werner Horsthemke. Effects of cross diffusion on Turing bifurcations in two-species reaction-transport systems. Phys. Rev. E, 83:036105, Mar 2011.

[20] Niraj Kumar, G.M. Viswanathan, and V.M. Kenkre. Hurst exponents for interacting random walkers obeying nonlinear Fokker-Planck equations. Physica A, 388(18):3687 3694, 2009.

[21] Attila Lazar, Horst-Dieter Forsterling, Andras Volford, and Zoltan Noszticzius. Refraction of chemical waves propagating in modified membranes. J. Chem. Soc., Faraday Trans., 92:2903-2909, 1996.

[22] W.-S. Li, W.-Y. Hu, Y.-C. Pang, T.-R. Liu, W.-R. Zhong, and Y.-Z. Shao. Regulation of turing patterns in a spatially extended chlorine-iodine-malonic-acid system with a local concentration-dependent diffusivity. Phys. Rev. E, 85(6), 2012.

[23] H.W. Liu, H.M. Guo, Y.L. Wang, Y.T. Wang, C.M. Shen, and L. Wei. Self-assembled stripes on the anodic aluminum oxide by atomic force microscope observation. Appl. Surf. Sci., 219(3-4):282-289, 2003.

[24] D. Mackey, L. Plantié, and M.M. Turner. Instabilities and pattern formation in low temperature plasmas. Appl. Math. Lett., 18(8):865 - 873, 2005.

[25] H. Malchow. Dissipative pattern formation in ternary non-linear reaction-electrodiffusion systems with concentration-dependent diffusivities. J. Theor. Biol., 135(3):371-381, 1988.

[26] G. Mulone, S. Rionero, and W. Wang. The effect of density-dependent dispersal on the stability of populations. Nonlinear Anal., 74(14):4831-4846, 2011.

[27] H. Nagashima. Target patterns and pacemakers in a reaction-diffusion system. J. Phys. Soc. Japan, 60(9):2797-2799, 1991.

[28] B. Neumann, Zs. Nagy-Ungvarai, and S.C. Muller. Interaction between silica gel matrices and the belousovzhabotinsky reaction. Chem. Phys. Lett., 211(1):36 - 40, 1993.

[29] Y. Pomeau, S. Zaleski, and P. Manneville. Axisymmetric cellular structures revisited. ZAMP, 36(3):367-394, 1985.

[30] C.J. Roussel and M.R. Roussel. Reaction-diffusion models of development with statedependent chemical diffusion coefficients. Prog. Biophys. Mol. Bio., 86(1):113-160, 2004. 
[31] M.R. Roussel and J. Wang. Transition from self-replicating behavior to stationary patterns induced by concentration-dependent diffusivities. Phys. Rev. Lett., 87(18):18830211883024, 2001.

[32] Ricardo Ruiz-Baier and Canrong Tian. Mathematical analysis and numerical simulation of pattern formation under cross-diffusion. Nonlinear Anal. Real World Appl., 14(1):601$612,2013$.

[33] Arnd Scheel. Radially symmetric patterns of reaction-diffusion systems. Mem. Amer. Math. Soc., 165(786):viii+86, 2003.

[34] J. A. Sherratt. Wavefront propagation in a competition equation with a new motility term modelling contact inhibition between cell populations. R. Soc. Lond. Proc. Ser. A Math. Phys. Eng. Sci., 456(2002):2365-2386, 2000.

[35] M. Stich and A. S. Mikhailov. Target patterns in two-dimensional heterogeneous oscillatory reactiondiffusion systems. Physica D, 215(1):38 - 45, 2006.

[36] Canrong Tian, Zhigui Lin, and Michael Pedersen. Instability induced by cross-diffusion in reaction-diffusion systems. Nonlinear Anal. Real World Appl., 11(2):1036-1045, 2010.

[37] A.E. Tikhomirova and V.A. Volpert. Nonlinear dynamics of endothelial cells. Appl. Math. Lett., 20(2):163 - 169, 2007.

[38] J. C. Tzou, A. Bayliss, B.J. Matkowsky, and V.A. Volpert. Interaction of Turing and Hopf modes in the superdiffusive Brusselator model near a codimension two bifurcation point. Math. Model. Nat. Phenom., 6:87-118, 02011.

[39] J.C. Tzou, B.J. Matkowsky, and V.A. Volpert. Interaction of Turing and Hopf modes in the superdiffusive Brusselator model. Appl. Math. Lett., 22(9):1432 - 1437, 2009.

[40] J. Wolff, M. Stich, C. Beta, and H. H. Rotermund. Laser-induced target patterns in the oscillatory co oxidation on pt(110). J. Phys. Chem. B, 108(38):14282-14291, 2004.

[41] E. P. Zemskov, K. Kassner, M. J. B. Hauser, and W. Horsthemke. Turing space in reaction-diffusion systems with density-dependent cross diffusion. Phys. Rev. E, 87:032906, Mar 2013.

[42] Evgeny P. Zemskov, Vladimir K. Vanag, and Irving R. Epstein. Amplitude equations for reaction-diffusion systems with cross diffusion. Phys. Rev. E, 84:036216, Sep 2011.

[43] C. Zhao, B.E. Hobbs, H.B. Mühlhaus, and A. Ord. Finite element modelling of dissipative structures for nonequilibrium chemical reactions in fluid-saturated porous media. Comput. Methods Appl. Mech. Engrg., 184(1):1-14, 2000.

[44] C. Zhao, B.E. Hobbs, H.B. Mühlhaus, A. Ord, and G. Lin. Computer simulations of coupled problems in geological and geochemical systems. Comput. Methods Appl. Mech. Engrg., 191(29-30):3137-3152, 2002. 